\title{
Managing pulmonary embolism using prognostic models: future concepts for primary care
}

\author{
Geert-Jan Geersing MD PhD, Ruud Oudega MD PhD, Arno W. Hoes MD PhD, Karel G.M. Moons PhD
}

$\mathrm{W}$ ith the availability of new treatment for thromboembolism that can be administered at home, the management of pulmonary embolism is changing. This has implications for both the location and provision of care. Pulmonary embolism occurs in 1-2 per 1000 people each year and in up to 10 per 1000 among elderly people. ${ }^{1,2}$

Diagnosing pulmonary embolism is sometimes difficult because the signs and symptoms can be subtle, and the results of diagnostic tests are not always conclusive. Thus, the diagnosis is usually made based on the results of multiple investigations, including clinical assessment, D-dimer testing and imaging (predominantly spiral computed tomography). ${ }^{3-5}$

After the diagnosis of pulmonary embolism is confirmed, which often happens in the emergency department, appropriate treatment must be started. Two important questions need to be answered: is there a need for admission to hospital, and for how long do patients need to take anticoagulants to minimize the risk of recurrence?

Answering these questions requires prognostic studies, which provide evidence of short-term (acute mortality) and long-term (recurrent events) risk. ${ }^{6,7}$ Given the availability of treatments that can be used in the outpatient setting and a growing body of literature about the safety of at-home treatment in specific groups of patients with pulmonary embolism, we anticipate this condition will be treated more and more by physicians who provide primary care (e.g., family physicians, general internists) in collaboration with relevant consultants.

In this article, we discuss the current models for predicting short- and long-term prognosis for patients with pulmonary embolism, with a focus on identifying patients at low risk of adverse outcomes. A summary of our literature review is available in Box 1. We defined short-term adverse outcomes as death caused by hemodynamic instability and shock. If death occurs, it is usually within one month of diagnosis and is often within one to two weeks. ${ }^{8}$ Long-term adverse outcomes include recurrence of pulmonary embolism, the risk of which can be as high as $10 \%$ per year. ${ }^{9}$

\section{Short-term prognosis}

In the past, patients with pulmonary embolism were admitted to hospital for at least five to seven days. The length of admission was related to the mode of administration of first-line treatment, which includes intravenously administered heparin, as well as to concerns about the high risk of death. Yet with the development of lowmolecular-weight heparin (administered subcutaneously) and, more recently, novel anticoagulants that can be taken orally, ${ }^{10,11}$ pulmonary embolism can now be treated on an outpatient basis. In addition, we now recognize that, if treatment is started quickly, pulmonary embolism does not always follow a dramatic course of hemodynamic instability and shock with subsequent risk of death. Thus, a key question is whether it is possible to determine which patients with pulmonary embolism have a low risk of death. Identifying these patients may provide an opportunity for outpatient treatment in a primary care setting.

\section{How can we identify patients with a low risk of death?}

Several clinical prediction models have been developed to identify patients with pulmonary embolism who have a low risk of death in the short term. ${ }^{12}$ In a prospective study involving 296 outpatients with pulmonary embolism, Wicki and colleagues identified six predictors of death (cancer, heart failure, previous deep vein thrombosis, systolic blood pressure $<100 \mathrm{~mm} \mathrm{Hg}$, arterial $\mathrm{PaO}_{2}<8 \mathrm{kPa}$, or the presence of deep vein thrombosis on ultrasound)
Competing interests: Geert-Jan Geersing, Ruud Oudega and Karel Moons have received unrestricted grants from GlaxoSmithKline, Inverness and Bayer Healthcare. No competing interests were declared by Arno Hoes.

This article has been peer reviewed.

Correspondence to: Dr. Geert-Jan Geersing, G.J.Geersing@umcutrecht.nl

CMAJ 2012. DOI:10.1503 /cmaj.110213

\footnotetext{
- Ker points

- Nearly half of all patients with pulmonary embolism are candidates for immediate discharge and subsequent outpatient care.

- Outpatient care may be supervised by primary care physicians, with vigilant attention given to those at risk of early treatment failure.

- The risk of recurrence of pulmonary embolism is low for provoked events and for unprovoked events with a normal D-dimer level after three to six months of treatment.

- In patients with a high risk of recurrence, this risk should be balanced with the risk of bleeding to decide on the optimal treatment duration; this risk assessment usually involves close collaboration with experts in this area.
} 
during a three-month follow-up period..$^{13}$ The resulting model, called the Geneva prediction rule, calculates a score by assigning two points each for the presence of cancer and hypotension, and one point each for all other variables. In their study group, about two-thirds of all patients had a score of two or less, which was associated with a $2 \%$ risk of adverse outcomes (repeat thrombotic event, major bleeding or death). This model relies on ultrasound findings, making it less useful in primary care, but it would be appropriate in most emergency department or hospital settings.

In contrast, the Pulmonary Embolism Severity Index (PESI) does not rely on ultrasound findings. Instead, it uses the patient's clinical history and the findings from a physical examination (Box 2). It is the most widely investigated model, with validation studies having been performed in European and North American populations. Because this model relies on items that are readily available in primary care settings, it is most suitable for use in an outpatient or primary care. The PESI model categorizes patients into five groups at different levels of adverse outcomes.

The results of 11 observational studies, $(n>$ 20000 patients) that used the PESI model to assess risk are summarized in Appendix 2 (available at www.cmaj.ca/lookup/suppl/doi:10.1503/ cmaj.110213/-/DC1). Using this model, 34\%-56\% of patients in the validation studies were classified as being at low risk (group I and II), with overall mortality of less than $2 \%$ and pulmonary embolism-specific mortality of less than $1 \%$.

The PESI model was recently simplified to enhance its clinical applicability. ${ }^{14}$ In the revised model, five variables have been removed. The six remaining variables in the simplified model are age greater than 80 years, history of cancer, chronic cardiopulmonary disease, pulse 110 beats/min or higher, systolic blood pressure less than 100 $\mathrm{mm} \mathrm{Hg}$, and oxygen saturation level less than $90 \%$. Each variable has a value of one point, and patients with no points (or the absence of all predictor variables) are classified as being at low-risk of adverse outcomes. In a validation cohort study involving

\section{Box 1: Summary of the literature review}

The goal of our review was to describe the available prognostic tools for treating pulmonary embolism in nonspecialized care; thus, we were interested in studies that evaluated prognostic tools available for use at this level of care. We searched MEDLINE and Embase (2001-June 2011) for English language articles using the terms "pulmonary embolism," "PESI," "Pulmonary Embolism Severity Index," "mortality," "VTE recurrence," "venous thromboembolism recurrence," and "prognosis." We searched the Cochrane database for systematic reviews. We searched the reference lists of identified studies. We also contacted experts in this area. A flowchart of our literature search is available in Appendix 1 (available at www.cmaj.ca /lookup/suppl/doi:10.1503/cmaj.110213/-/DC1).
7106 patients with pulmonary embolism, the simplified model identified $36 \%$ of patients as being at low risk, with an overall mortality of $1.1 \%$ (95\% confidence interval $[\mathrm{CI}] 0.7 \%-1.5 \%){ }^{6}$

\section{Is outpatient treatment safe for patients with pulmonary embolism?}

In 2008 and 2009, two systematic reviews ${ }^{15,16}$ were published about the safety of outpatient management of pulmonary embolism in patients at low-risk of short-term complications. The 2008 review by Janjua and colleagues was based on six observational studies, with a total of 593 outpatients. ${ }^{15}$ The 2009 review by Squizzato and colleagues included the same six studies plus five additional observational studies, giving a total of 928 patients with pulmonary embolism. ${ }^{16}$ The 11 included studies did not use the PESI model to identify patients at low-risk of adverse outcomes. Instead, they relied on criteria related to hemodynamic instability and hypoxia. The short-term prognosis in patients who received outpatient care was excellent: no fatal pulmonary emboli occurred during 7-10 days of follow-up. However, both reviews stressed that their results were based on small studies, some of which were of low methodologic quality (e.g., lack of standardized risk-assessment methods, different treatment

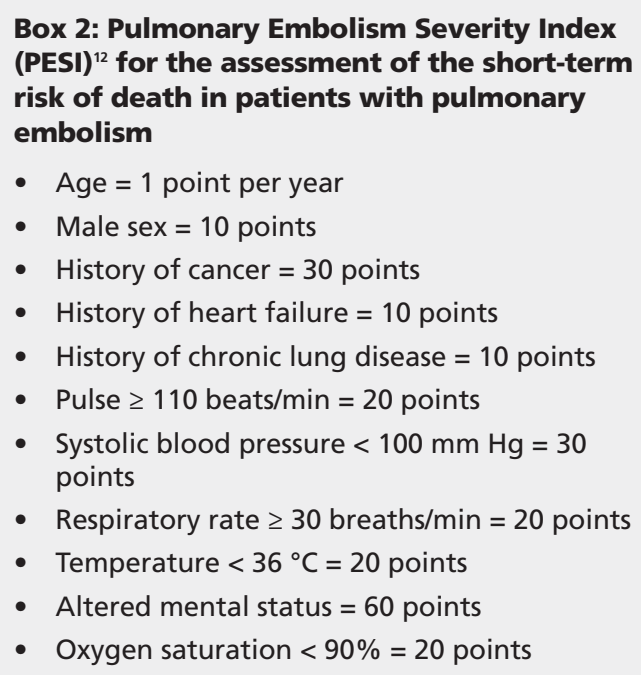

Group I (low risk): $\leq 65$ points; group II (low risk): 66-85 points; group III: 86-105 points; group IV: 106-125 points; group $V>125$ points (the risk in groups III-V is categorized as not low)

Patients in group I and II are considered to be at low risk of death in the short term $(<2 \%)$. For example, a 40-year-old man with no comorbidities, no hemodynamic instability, no altered mental status, a normal temperature and an oxygen saturation level of $98 \%$ would have a PESI score of 50 points (group I). Thus, the risk of death in the short term is predicted to be low. 
regimes in different studies, and a lack of randomized studies).

Since these reviews were performed, five additional studies have been published: three observational cohort studies ${ }^{17-19}$ and two randomized controlled trials..$^{20,21}$ In the three observational studies, which included about 750 patients with pulmonary embolism who received outpatient care, there was only one death (because of metastatic cancer). In contrast, the trial by Otero and colleagues was stopped prematurely. ${ }^{21}$ After randomly assigning 132 patients at low-risk of death in the short term to in- or outpatient care, there was $2.8 \%(95 \% \mathrm{CI}$ $0.8 \%-9.6 \%$ ) mortality in the first 10 days among patients who received outpatient care. There were no deaths among patients receiving inpatient care. This increased mortality, as well as the associated causes (one fatal pulmonary embolism and one fatal gastrointestinal hemorrhage) prompted the data-safety monitoring board to stop the study.

In the randomized controlled trial by Aujesky and colleagues, the PESI model was used to identify patients at low risk of death in the short term..$^{20}$ Patients at low risk and who met the inclusion criteria (e.g., absence of hypoxia, hemodynamic instability or contraindications for anticoagulant treatment) were randomly assigned to either in- or outpatient care $(n=344)$. The mortality rate $(0.6 \%)$ was not different in either group.

The conflicting results from these two trials seem to be primarily related to the selection criteria used to identify patients at low-risk. Otero and colleagues ${ }^{21}$ did not use a validated risk assessment tool, such as the PESI model, which was used by Aujesky and colleagues. ${ }^{20}$ Indeed, $55 \%$ of patients were found to be suitable for outpatient care in the trial by Otero and colleagues, whereas $30 \%$ of patients received outpatient care in the trial by Aujesky and colleagues, indicating that more stringent criteria were used in the latter trial.

\section{How can prognostic scores for short-term risk guide practice?}

After the diagnosis of pulmonary embolism is confirmed, the risk of death in the short term should be assessed. Based on current evidence, the best approach is to calculate the patient's PESI score. Patients with a PESI score of 85 points or less (group I or II; Box 2) are candidates for outpatient care, provided that they do not have hypoxia, hemodynamic instability or contraindications to anticoagulant therapy. After early discharge (within 24 hours if possible) from the emergency department or hospital, patients at low risk of death in the short term can be cared for by their primary care physician. This will usually include (a) ensuring that low-molecular-weight heparin is given subcutaneously for at least five days, (b) management of international normalized ratio in patients receiving vitamin $\mathrm{K}$ antagonists, and (c) early recognition of possible treatment failure (i.e., recurrent pulmonary embolism or bleeding, with subsequent readmission to the hospital if needed.

Douketis and colleagues studied the determinants of treatment failure in a cohort of 1021 patients with deep vein thrombosis or pulmonary embolism. ${ }^{22}$ During the first three months of treatment, $28(3 \%)$ patients experienced major bleeding and $58(6 \%)$ patients had a recurrent event while taking an anticoagulant. These treatment failures predominantly occurred during the first weeks after treatment was started: $75 \%(21 / 28)$ of major bleeding complications and $72 \%(42 / 58)$ of recurrent events occurred within three weeks. In their multivariable analysis, Douketis and colleagues found that cancer, chronic cardiovascular disease, chronic respiratory disease and other concurrent disease (renal, hepatic, gastrointestinal, neurologic, hematologic or multisystem disease) were independently associated with an increased risk of recurrent disease while receiving anticoagulant therapy. Therefore, primary care physicians should be particularly vigilant for treatment failures in patients with these conditions, especially during the first three weeks after starting treatment.

\section{Long-term prognosis}

Whereas the initial goal of starting anticoagulant treatment in patients with pulmonary embolism is minimizing the short-term risk of death, ongoing treatment should be aimed at minimizing the risk of recurrence. While receiving anticoagulant treatment, the risk of recurrence of pulmonary embolism is low (less than $1 \%$ per year $\left.{ }^{23}\right)$. After treatment is stopped, the risk rapidly increases (especially in the first months after treatment is stopped) to as high as $10 \%$ in the first year and more than $30 \%-40 \%$ within five years. ${ }^{24}$ Yet, continuing anticoagulant treatment also increases the risk of bleeding by threefold to about $1 \%$ per year from a $0.3 \%$ risk while not taking anticoagulants. ${ }^{23}$

Currently, most guidelines (e.g., the antithrombotic and antihemolytic therapy guidelines by the American College of Chest Physicians ${ }^{9}$ ) recommend balancing the risk of bleeding with the risk of recurrence after an initial treatment period of three to six months. Recently developed prognostic tools ${ }^{25}$ can help physicians to objectively identify which patients have the highest risk of recurrence. These tools can also help physicians decide how long a patient with a confirmed first episode of pulmonary embolism should receive anticoagulant therapy. 


\section{How can patients with a low risk of recurrence be identified?}

Prognostic indicators, such as the presence of D-dimer, can be helpful in predicting long-term prognosis for patients with pulmonary embolism. Long-term prognosis differs for patients with provoked (e.g., by a transient risk factor such as recent surgery) or an unprovoked (i.e., idiopathic or spontaneous) pulmonary embolism. A recent metaanalysi ${ }^{26}$ showed that patients with a provoked event (defined by the authors as being related to recent surgery, immobilization, puerperium, estrogen use, major trauma or medical illness have a low risk of recurrence. The risk of recurrence has been reported to be less than $1 \%$ for provoked pulmonary embolism caused by surgical factors and about $4 \%$ for those caused by nonsurgical factors (e.g., medical illness or immobilization). ${ }^{26}$

In contrast, patients with an unprovoked pulmonary embolism generally have a higher risk of recurrence than patients with provoked pulmonary embolism. ${ }^{27}$ However, this group of patients is heterogeneous and can range, for example, from a woman with a first pulmonary embolism at age 34 years to a man with a first episode at 71 years. For some patients, it is unclear whether an event was provoked or not (e.g., a woman with a pulmonary embolism who is using estrogen. ${ }^{28-30}$ Such patients can be placed in either group (provoked or unprovoked). Therefore, further risk stratification is needed in this group because of the heterogeneous patient population and uncertainty about whether an event is provoked or not.

Recent studies have suggested that D-dimer testing after three to six months of treatment can help to identify patients with unprovoked pulmonary embolism who have a low risk of recurrence (similar to the low risk of recurrence associated with provoked events). D-dimer testing is usually performed three to five weeks after stopping anticoagulation treatment. A landmark study in this area, the PROLONG study, was published in 2006. ${ }^{31}$ This study randomly assigned 227 patients with unprovoked deep vein thrombosis, pulmonary embolism and elevated D-dimer levels after an initial treatment period with anticoagulants to either stop or resume anticoagulant treatment. Among patients who stopped treatment, the annualized incidence of recurrence was $11 \%$ (18 events per 120 patients), while the rate was $2 \%$ among patients who resumed treatment ( 3 events per 103 people).

The higher risk of recurrence among patients with unprovoked pulmonary embolism and an elevated D-dimer level was later confirmed in a meta-analysis by Verhovsek and colleagues. ${ }^{32}$ This analysis included the PROLONG study and six additional studies involving 1888 patients.
Among patients with normal D-dimer levels after treatment ( $n=981)$, the annual rate of recurrence of deep venous thrombosis or pulmonary embolism was $3.5 \%$ (95\% CI 2.7\%-4.3\%).

A recent meta-analysis using individual patient data from the same seven studies confirmed the robustness of this finding. ${ }^{33}$ The association between elevated D-dimer levels and risk of recurrence was not influenced by patient age, cut-off point of the D-dimer assay or the timing of D-dimer testing (whether in the first three weeks after stopping treatment, three to five weeks after stopping or more than five weeks after stopping). ${ }^{33}$ The only variable that had an additional effect on the risk of recurrence was male sex, with a higher risk among men. This was later confirmed by Eichinger and colleagues, who developed (and internally validated using bootstrapping techniques) a prognostic model based on data from 929 patients with unprovoked deep vein thrombosis or pulmonary embolism (the Vienna model)..$^{34}$ Male sex was included in their model, as well as the type of event (pulmonary embolism $\mathrm{v}$. deep vein thrombosis) and D-dimer levels (Box 3).

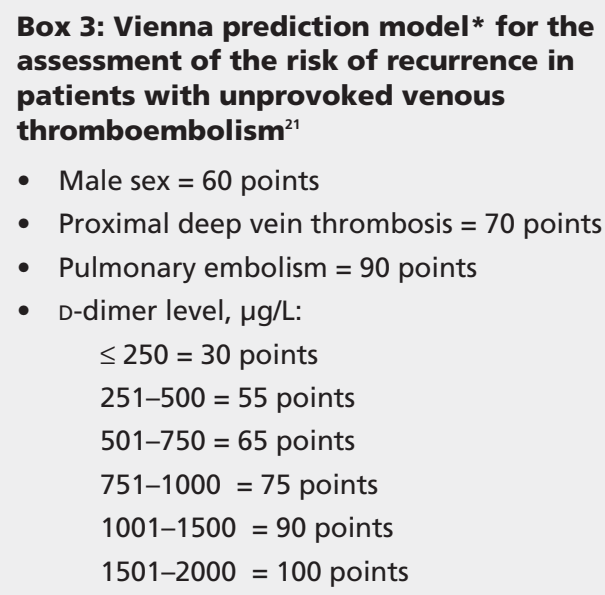

- Male sex $=60$ points

- Proximal deep vein thrombosis $=70$ points

- Pulmonary embolism $=90$ points

- D-dimer level, $\mu \mathrm{g} / \mathrm{L}$ :

$\leq 250=30$ points

$251-500=55$ points

$501-750=65$ points

$751-1000=75$ points

$1001-1500=90$ points

$1501-2000=100$ points

Low risk $\leq 170$ points; moderate risk 171-240 points; high risk $>240$ points.

For patients at low risk, the expected rate of recurrent venous thromboembolism is below $5 \%$ at one year. The recurrence rate for those at moderate risk is $5 \%-10 \%$ at one year and the rate for those at high risk is greater than $10 \%$. For example, a male patient with an unprovoked pulmonary embolism and a D-dimer level of about $1000 \mu \mathrm{g} / \mathrm{L}$ after three to six months of treatment has a Vienna score of 225. Thus, expected risk of recurrence for this patient at one year is between $5 \%$ and $10 \%$.

*The original Vienna model incorporates

D-dimer results on a continuous scale. An online prediction calculator for this model is available in Appendix 3 (available at www.cmaj.ca/lookup/suppl/doi:10.1503/cmaj 110213/-/DC1) 
The REVERSE study found that male sex was an independent predictor of recurrence. ${ }^{35}$ This study included 648 patients with unprovoked deep vein thrombosis or pulmonary embolism (600 patients completed a mean follow-up of 18 months), and the authors assessed 69 potential predictors of recurrence in a multivariable analysis. They concluded that all male patients had a high risk of recurrence, as did women with two or more of the following risk factors: hyperpigmentation, edema or redness of either leg, elevated D-dimer levels ( $250 \mu / \mathrm{L})$ while taking anticoagulants, body mass index of 30 or higher, or aged 65 years or older.

Additional resources are provided in Appendix 3 (available at www.cmaj.ca/lookup/suppl /doi:10.1503/cmaj.110213/-/DC1).

\section{How can prognostic indicators for long- term risk guide practice?}

Prognostic indicators for the risk of recurrence can help physicians determine the optimal duration of anticoagulant treatment for patients with pulmonary embolism. In patients with provoked events, as well as patients with unprovoked events and a low risk of recurrence (as indicated by normal D-dimer levels or a low score using the Vienna or REVERSE model), the annual risk of recurrence of about $2 \%-4 \%$ does not outweigh the $1 \%$ per year risk of major bleeding complications associated with prolonged treatment. ${ }^{36}$ Thus, these patients could receive treatment for a fixed duration, for example, of three to six months.

Patients with a higher risk of recurrence (e.g., those with an unprovoked event in combination with elevated D-dimer levels after treatment is stopped or a high score on the Vienna or REVERSE model) may benefit from continuing, perhaps indefinitely, anticoagulant treatment. Because prolonging treatment should be balanced with the risk of bleeding, it should only be considered for patients who have shown good tolerance and compliance to anticoagulants or who have a low risk of bleeding complications (e.g., no renal or hepatic failure, or age below an arbitrarily chosen threshold of 75 years)..$^{27}$

Risk stratification helps to objectively determine the risk of recurrence. As well, it provides guidance to policy and guideline makers in deciding who should be responsible for supervising the care of patients with pulmonary embolism. Patients with a low risk of recurrence for whom a fixed duration of treatment is advocated (based on this low risk of recurrence) could safely receive treatment in a primary care setting. Patients for whom prolonged treatment is considered necessary given their high risk of recurrence could receive care entirely in a secondary care setting or in primary care with close collaboration with relevant consultants. Box 4 provides a fictional example of how prognostic models can be used in clinical practice.

\section{Gaps in knowledge}

There is ongoing research and unanswered questions remain about the use of prognostic models in pulmonary embolism. We suggest that the value of adding cardiac markers, such as brain natriuretic peptide and troponin, ${ }^{37,38}$ to the PESI model should be investigated. One study reported that adding troponin to the PESI model had a modest added value, ${ }^{39}$ but such studies for brain natriuretic peptide are currently lacking.

Predicting recurrence of venous thromboembolism in patients with pulmonary embolism seems to be feasible based on the results of D-dimer testing. However, the impact of D-dimer testing on patient care has been evaluated in only one relatively small randomized controlled trial. ${ }^{31}$ More studies, either observational or, preferably, larger randomized controlled trials, are needed. Currently three observational studies (NCT00720915,

\section{Box 4: Applying the results of this review in clinical practice} (fictional case)

A 68-year-old woman with chronic obstructive pulmonary disease presents to a family medicine clinic with a one-day history of shortness of breath and pain on inspiration. On physical examination, no signs of an exacerbation of chronic obstructive pulmonary disease (e.g., fever, or rales or rhonchi on auscultation) are found. Her oxygen saturation is $88 \%$ on pulse oximetry, and she has tachycardia (120 beats/min) with a systolic blood pressure of 120 $\mathrm{mm} \mathrm{Hg}$. Because pulmonary embolism cannot be ruled out, she is referred to the emergency department for further assessment. Spiral computed tomography confirms the presence of pulmonary embolism, and anticoagulant treatment is started. Should she be admitted to hospital? For how long should she be given anticoagulants?

Using the PESI model, this patient has a score of 118 (68 points for age, 10 for history of chronic lung disease, 20 for tachycardia and 20 for lowered oxygen saturation level). She is thus in PESI group IV, and should be admitted to hospital for treatment, given her elevated risk of death. However, if she had no history of COPD, no lowered oxygen saturation and no tachycardia, her PESI score would be 68 points (for age). This patient would be in PESI group II, and she would be a candidate for outpatient treatment of pulmonary embolism, supervised by her primary care physician.

After taking anticoagulants for six months, the patient's risk of long-term recurrence can be assessed via D-dimer testing four weeks after stopping anticoagulants. According to the Vienna model, if her D-dimer level is around $1000 \mu \mathrm{g} / \mathrm{L}$, the predicted risk of recurrence is about $5 \%$ in the first year (score of $180=$ moderate risk). If, however, her D-dimer level is $250 \mu \mathrm{g} / \mathrm{L}$, the risk is predicted to be around $3 \%$ (score of $120=$ low risk). If she has good tolerance and compliance with anticoagulant treatment and is found to be at high risk, long-term treatment might be warranted. In contrast, if her risk is predicted to be low, she may be a candidate for stopping anticoagulant treatment.

If the patient has an elevated D-dimer level, collaboration with a thrombosis specialist can be sought to balance the increased risk of recurrent pulmonary embolism with the increased risk of bleeding associated with long-term anticoagulant treatment. If a decision is made to continue anticoagulant treatment indefinitely, the risks should be reassessed yearly. 
NCT00954395 and NCT00967304) and one randomized controlled trial (NTR2680) are recruiting patients. In addition, the impact of patient sex and type of venous thromboembolism (as included in the Vienna model) on the results of D-dimer testing should be further investigated. Finally, the applicability and validity of using both the PESI and Vienna models in primary care should be further investigated. Additional details of ongoing research and knowledge gaps are presented in Appendix 4 (available at www.cmaj.ca/lookup/suppl/doi :10.1503/cmaj.110213/-/DC1).

\section{References}

1. Chunilal SD, Eikelboom JW, Attia J, et al. Does this patient have pulmonary embolism? JAMA 2003;290:2849-58.

2. Siccama RN, Janssen KJ, Verheijden NA, et al. Systematic review: diagnostic accuracy of clinical decision rules for venous thromboembolism in elderly. Ageing Res Rev 2011;10:304-13.

3. Kearon C. Diagnosis of pulmonary embolism. CMAJ 2003;168: 183-94.

4. Qaseem A, Snow V, Barry P, et al. Current diagnosis of venous thromboembolism in primary care: a clinical practice guideline from the American Academy of Family Physicians and the American College of Physicians. Ann Fam Med 2007;5:57-62.

5. Stein PD, Fowler SE, Goodman LR, et al. Multidetector computed tomography for acute pulmonary embolism. $N$ Engl J Med 2006;354:2317-27.

6. Moons KG, Royston P, Vergouwe Y, et al. Prognosis and prognostic research: What, why, and how? BMJ 2009;338:b375.

7. Royston P, Moons KG, Altman DG, et al. Prognosis and prognostic research: developing a prognostic model. BMJ 2009;338:b604

8. Kearon C. Natural history of venous thromboembolism. Circulation 2003;107(Suppl 1):I22-30.

9. Kearon C, Kahn SR, Agnelli G, et al. Antithrombotic therapy for venous thromboembolic disease: American College of Chest Physicians evidence-based clinical practice guidelines (8th edition). Chest 2008;133(6 Suppl):454S-545S.

10. Bauersachs R, Berkowitz SD, Brenner B, et al. Oral rivaroxaban for symptomatic venous thromboembolism. N Engl J Med 2010; 363:2499-510.

11. Schulman S, Kearon C, Kakkar AK, et al. Dabigatran versus warfarin in the treatment of acute venous thromboembolism. $N$ Engl J Med 2009;361:2342-52.

12. Jiménez D, Yusen RD, Otero R, et al. Prognostic models for selecting patients with acute pulmonary embolism for initial outpatient therapy. Chest 2007;132:24-30.

13. Wicki J, Perrier A, Perneger TV, et al. Predicting adverse outcome in patients with acute pulmonary embolism: a risk score. Thromb Haemost 2000;84:548-52.

14. Jiménez D, Aujesky D, Moores L, et al. Simplification of the pulmonary embolism severity index for prognostication in patients with acute symptomatic pulmonary embolism. Arch Intern Med 2010;170:1383-9.

15. Janjua M, Badshah A, Matta F, et al. Treatment of acute pulmonary embolism as outpatients or following early discharge. A systematic review. Thromb Haemost 2008;100:756-61.

16. Squizzato A, Galli M, Dentali F, et al. Outpatient treatment and early discharge of symptomatic pulmonary embolism: a systematic review. Eur Respir J 2009;33:1148-55.

17. Agterof MJ, Schutgens RE, Snijder RJ, et al. Out of hospital treatment of acute pulmonary embolism in patients with a low NT-proBNP level. J Thromb Haemost 2010;8:1235-41.

18. Erkens PM, Gandara E, Wells P, et al. Safety of outpatient treatment in acute pulmonary embolism. J Thromb Haemost 2010;8:2412-7.

19. Kovacs MJ, Hawel JD, Rekman JF, et al. Ambulatory management of pulmonary embolism: a pragmatic evaluation. J Thromb Haemost 2010;8:2406-11.

20. Aujesky D, Roy PM, Verschuren F, et al. Outpatient versus inpatient treatment for patients with acute pulmonary embolism: an international, open-label, randomised, non-inferiority trial. Lancet 2011;378:41-8.

21. Otero R, Uresandi F, Jimenez D, et al. Home treatment in pulmonary embolism. Thromb Res 2010;126:e1-5.
22. Douketis JD, Foster GA, Crowther MA, et al. Clinical risk factors and timing of recurrent venous thromboembolism during the initial 3 months of anticoagulant therapy. Arch Intern Med 2000;160:3431-6.

23. Hutten BA, Prins MH. Duration of treatment with vitamin K antagonists in symptomatic venous thromboembolism. Cochrane Database Syst Rev 2006;(1):CD001367.

24. van Dongen CJ, Vink R, Hutten BA, et al. The incidence of recurrent venous thromboembolism after treatment with vitamin $\mathrm{K}$ antagonists in relation to time since first event: a meta-analysis. Arch Intern Med 2003;163:1285-93.

25. Kyrle PA, Rosendaal FR, Eichinger S. Risk assessment for recurrent venous thrombosis. Lancet 2010;376:2032-9.

26. Iorio A, Kearon C, Filippucci E, et al. Risk of recurrence after a first episode of symptomatic venous thromboembolism provoked by a transient risk factor: a systematic review. Arch Intern Med 2010;170:1710-6.

27. Kearon C. Balancing risks and benefits of extended anticoagulant therapy for idiopathic venous thrombosis. J Thromb Haemost 2009;7(Suppl 1):296-300.

28. Kyrle PA, Minar E, Bialonczyk C, et al. The risk of recurrent venous thromboembolism in men and women. N Engl J Med 2004; 350:2558-63.

29. Le Gal G, Kovacs MJ, Carrier M, et al. Risk of recurrent venous thromboembolism after a first oestrogen-associated episode. Data from the REVERSE cohort study. Thromb Haemost 2010; 104:498-503.

30. McRae S, Tran H, Schulman S, et al. Effect of patient's sex on risk of recurrent venous thromboembolism: a meta-analysis. Lancet 2006;368:371-8.

31. Palareti G, Cosmi B, Legnani C, et al. D-dimer testing to determine the duration of anticoagulation therapy. N Engl J Med 2006; 355:1780-9

32. Verhovsek M, Douketis JD, Yi Q, et al. Systematic review: D-dimer to predict recurrent disease after stopping anticoagulant therapy for unprovoked venous thromboembolism. Ann Intern Med 2008;149:481-90, W94.

33. Douketis J, Tosetto A, Marcucci M, et al. Patient-level meta-analysis: effect of measurement timing, threshold, and patient age on ability of D-dimer testing to assess recurrence risk after unprovoked venous thromboembolism. Ann Intern Med 2010;153:523-31.

34. Eichinger S, Heinze G, Jandeck LM, et al. Risk assessment of recurrence in patients with unprovoked deep vein thrombosis or pulmonary embolism: the Vienna prediction model. Circulation 2010;121:1630-6.

35. Rodger MA, Kahn SR, Wells PS, et al. Identifying unprovoked thromboembolism patients at low risk for recurrence who can discontinue anticoagulant therapy. CMAJ 2008;179:417-26.

36. Kearon C, Iorio A, Palareti G. Risk of recurrent venous thromboembolism after stopping treatment in cohort studies: recommendation for acceptable rates and standardized reporting. sJ Thromb Haemost 2010;8:2313-5.

37. Becattini C, Vedovati MC, Agnelli G. Prognostic value of troponins in acute pulmonary embolism: a meta-analysis. Circulation 2007;116:427-33.

38. Klok FA, Mos IC, Huisman MV. Brain-type natriuretic peptide levels in the prediction of adverse outcome in patients with pulmonary embolism: a systematic review and meta-analysis. Am J Respir Crit Care Med 2008;178:425-30.

39. Singanayagam A, Scally C, Al-Khairalla MZ, et al. Are biomarkers additive to pulmonary embolism severity index for severity assessment in normotensive patients with acute pulmonary embolism? QJM 2011;104:125-31.

Affiliations: From the Julius Center for Health Sciences and Primary Care, University Medical Center Utrecht, Utrecht, the Netherlands.

Contributors: Geert-Jan Geersing and Ruud Oudega conceived the article. Geert-Jan Geersing drafted the article, which was revised by all authors. All of the authors approved the final version submitted for publication.

Acknowledgements: Each of the authors has received grant funding from the Netherlands Heart Foundation. The authors are supported by the Netherlands Organisation for Scientific Research (ZonMW 945-04-009) and the Netherlands Heart Foundation (project no. 2006B236). These organizations had no influence on any aspect of this review. 